\title{
Nouveau cursus en radioprotection médicale destiné aux médecins, avec option d'un enseignement en ligne (e-learning)
}

\author{
Marc Otto ${ }^{a}$, \\ Jean-François Valley ${ }^{b}$, \\ Max Giger \\ a pnn ag \\ b ancien directeur IRA, Lausanne \\ c Président de la CFPC, \\ membre du Comité central \\ de la FMH
}

\begin{abstract}
Résumé
Compte tenu des expériences faites lors des cours de radioprotection pour médecins entre 1994 et 2004, l'Office fédéral de la santé publique (OFSP) et la Fédération des médecins suisses (FMH) ont convenu d'adopter une nouvelle pondération des objectifs de formation et de les unifier. L'enseignement permettant d'acquérir la «qualification technique» et la «qualité d'expert» sera à l'avenir destiné à tous les médecins exerçant une activité radiologique, que ce soit dans une institution ou de manière indépendante. Les contenus théoriques de la formation postgraduée en radioprotection peuvent désormais être acquis dans des cours ex cathedra ou à distance (e-learning) et les contenus pratiques dans un laboratoire à l'aide de modèles. Selon le titre de spécialiste visé, il est donné plus d'importance à la radioscopie ou à la radiographie. Cette formation postgraduée s'achève par un examen unique sous forme de questionnaire à choix multiple. La création d'un enseignement à distance (e-learning) permet de réduire à deux jours la présence au cours. La nouvelle plate-forme centrale d'inscription www.radioprotection.ch donne des renseignements sur l'ensemble des cours offerts en Suisse et permet aux médecins de s'inscrire en ligne au cours de leur choix.

L'enseignement des contenus cliniques et spécialisés reste l'affaire des établissements de formation postgraduée médicale reconnus par la FMH.
\end{abstract}

\section{Introduction}

La FMH et l'OFSP en tant qu'autorité de surveillance ont convenu de réaliser un projet commun pour transmettre la théorie de la radioprotection par un enseignement en ligne (e-learning). L'OFSP a chargé un physicien médical expérimenté en radioprotection et techniques radiologiques d'élaborer la matière d'enseignement. La mise en œuvre a été confiée à pnn ag, une société «spin-off» issue de l'EPF de Zurich. Un comité consultatif a été créé pour le pilotage du projet, notamment pour le développement et la gestion des contenus. Il se compose d'un délégué de la Société suisse de radiologie médicale et d'un délégué de la Société suisse de médecine générale ainsi que d'un physicien médical. La FMH et l'OFSP ont été invités en tant qu'hôtes permanents. Le degré d'importance et le classement des objectifs de formation ont été fixés par un autre organe dans lequel étaient représentés, la radiologie, la médecine générale et la physique médicale, ainsi que l'Association suisse des médecinsassistants et chefs de clinique (ASMAC), l'OFSP et la FMH.

\section{Enseignement}

L'enseignement théorique et pratique est basé sur la législation en matière de radioprotection et se compose de quatre parties théoriques et d'une partie pratique.

\section{Bases de la radioprotection}

- Rayons ionisants;

- interaction du rayonnement avec la matière;

- action biologique;

- principes de la radioprotection.

\section{Technique radiologique}

- Fonctionnement d'une installation radiologique;

- interaction du rayonnement avec le patient;

- détection de la radiation;

- modalités d'utilisation des installations radiologiques.

\section{Mesures de radioprotection}

- Détermination de la dose au patient;

- qualité de l'image en radiologie;

- optimisation de la dose au patient;

- protection des personnes exposées aux rayonnements dans l'exercice de leur profession.

\section{Législation en matière de radioprotection}

- Législation sur la radioprotection;

- régime de l'autorisation et rôle de l'expert;

- assurance de la qualité dans le radiodiagnostic;

- niveaux de référence diagnostiques.

\section{Partie pratique}

- Fonctionnement des installations radiologiques;

- dosimétrie individuelle et technique de réglage; 
- méthodes pratiques pour minimiser l'exposition aux rayonnements du patient et de l'opérateur.

\section{Examen en ligne}

Un catalogue détaillé des objectifs de formation ayant vu le jour dans la foulée de l'introduction de l'enseignement en ligne, les centres de formation en radioprotection médicale pour médecins reconnus par l'OFSP ont décidé d'instaurer un processus d'examen unifié sanctionnant ce cursus. Une commission d'examen a été chargée de surveiller cet examen et d'en gérer le pool de questions; chaque centre de formation y est représenté par un délégué.

Un schéma directeur et un pool de questions à choix multiple ont été développés pour l'examen sur la base du catalogue des objectifs de formation. L'examen consiste en une sélection individuelle de questions établie pour chaque candidat; à l'aide du schéma directeur, on s'assure que la pondération des objectifs examinés et la part des divers types de questions utilisés restent les mêmes.

La participation à l'examen est ouverte à chaque candidat ayant suivi avec succès la théorie et la pratique. L'examen consiste en 32 questions à choix multiple (de type A+, A- et K-prime) auxquels le candidat doit répondre en $60 \mathrm{mi}-$ nutes. L'examen est réussi à partir de 21 réponses correctes au minimum. En cas d'échec, le candidat peut se représenter. Sont admis comme documents pendant l'examen uniquement les bases légales (loi sur la radioprotection et ordonnances correspondantes).

Jusqu'à présent, les examens ont été réalisés directement à la suite de la partie pratique, dans un local équipé d'ordinateurs. Comme il est impossible, lors d'examens en ligne, d'identifier les candidats de façon fiable par voie automatisée, le centre de formation qui organise l'examen doit attester que c'est effectivement le candidat

\footnotetext{
Inscription sous www.radioprotection.ch

Afin de simplifier le processus d'inscription à un cours de radioprotection, les quatre centres de formation reconnus, soit I'Institut Paul-Scherrer PSI, I'Institut de radiophysique appliquée IRA ainsi que les entreprises SAFPRO et pnn ag, ont instauré un site internet commun (www.radioprotection.ch) à I'intention des médecins concernés. $Y$ sont répertoriés tous les cours de radioprotection sous forme de «paquets de formation» comprenant la théorie, la pratique et l'examen final d'une part, ainsi que les informations nécessaires (lieu, heures, coûts) et les places disponibles d'autre part. Dès que le centre de formation concerné a validé une inscription, celle-ci est confirmée par courriel. A partir de cet instant, chaque participant peut consulter son programme individuel au moyen d'un mot de passe.
}

inscrit qui passe son examen sur l'ordinateur. Les réponses sont ensuite immédiatement corrigées et évaluées. L'OFSP a chargé la firme pnn ag d'organiser cet examen en ligne.

\section{Premières expériences avec le nouveau cursus}

Jusqu'à fin août 2007, 14 cours ont été suivis par plus de 140 médecins en tout, dont env. $40 \%$ se sont inscrits par la plate-forme en ligne commune www.radioprotection.ch. Tous les examens ont été organisés et les certificats finaux (décisions) établis par le biais du nouveau système en ligne.

La simplification et l'uniformisation du catalogue des objectifs de formation ainsi que l'examen structuré sanctionnant le cours ont entraîné une augmentation des exigences, aussi bien pour les centres de formation que pour les candidats.

Vu l'enseignement très dense dispensé lors de la partie théorique, un nouveau déroulement du cours est en cours d'expérimentation. On veillera, surtout lors de cours organisés sur quatre jours consécutifs, à proposer une alternance de blocs théoriques brefs et de pratique. Les unités théoriques devraient s'en trouver allégées et le cours plus varié.

S'agissant de l'enseignement en ligne (e-learning), de petites adaptations ont été réalisées pour mieux guider les utilisateurs, et ce sont surtout les éléments servant à répéter la matière et à préparer l'examen qui ont été développés et élargis.

La Commission d'examen n'a dû traiter aucun recours jusqu'à présent. Durant la phase de démarrage du nouveau concept, l'ensemble des examens non réussis sont contrôlés, un à un, par des experts. En outre, toutes les questions posées sont analysées sur la base d'une évaluation statistique des réponses données lors de l'examen, puis discutées au cours des séances de la Commission d'examen. Cette dernière a ainsi déjà pu affiner le pool de questions lors d'une première séance.

\section{Enseignement en ligne (e-learning)}

L'enseignement en ligne se fonde sur une structure détaillée et systématique, selon laquelle le cours est subdivisé en 16 leçons composées de 10 chapitres chacune. Chacun des 160 chapitres porte sur des objectifs de formation spécifiques. Ces chapitres sont automatiquement configurés selon le type de cours (radiographie, radioscopie). Le candidat doit avoir travaillé sur tous les chapitres obligatoires pour obtenir l'attestation qui certifie la fréquentation réussie du cours 


\section{Radioprotection médicale: cursus actuel destiné aux médecins}

Les nouveaux cours de radioprotection pour médecins sont organisés depuis le début 2007. Les médecins qui souhaitent déposer une demande d'autorisation pour l'exploitation d'une installation radiologique, de même que ceux qui visent un titre de spécialiste dont le programme de formation postgraduée exige une formation en radioprotection, doivent obligatoirement accomplir ce cursus avec succès.

Le cours de radioprotection est offert en deux variantes: technique radiographique conventionnelle (type A) et radioscopie (type B). Le cursus est composé d'une partie théorique et d'une partie pratique de deux jours de formation chacune et d'un examen final standardisé de questions à choix multiple. Le candidat peut acquérir la partie théorique sous forme traditionnelle en fréquentant les deux jours de cours ou sous forme d'enseignement à distance par internet, à un moment et en un lieu de son choix. La partie pratique doit impérativement être suivie sur place dans un institut de formation reconnu, car le candidat peut y travailler sur des appareils de radiologie et passer l'examen QCM sur un ordinateur de l'institut concerné.

donné en ligne. Cette attestation permet de participer directement aux jours de pratique, puis de se présenter à l'examen. L'ordre dans lequel les 16 leçons doivent être traitées est partiellement prescrit. La première leçon consacrée aux bases physiques des rayonnements ionisants, ainsi que les quatre leçons relatives à la législation sur la radioprotection, peuvent être étudiées dans un ordre librement choisi. Les 11 autres leçons, consacrées aux bases physiques, aux installations radiologiques et aux aspects de radioprotection médicale concernant les patients, ont une structure progressive et doivent par conséquent être étudiées dans l'ordre donné.

Chaque chapitre est structuré de la même façon et contient les mêmes éléments: l'exposé du problème (objectif de formation), suivi d'un résumé répondant à la question posée et de contenus de formation développant en détail les énoncés du résumé. Des exercices, des animations et des informations complémentaires permettent d'approfondir les divers contenus. Chaque chapitre contient plusieurs questions de contrôle donnant au candidat la possibilité de vérifier les progrès accomplis.

Pour profiter d'un enseignement individuel, les apprenants peuvent choisir entre trois modes d'étude. Ces modes se distinguent entre eux par le choix et l'ordre des éléments. Dans le «mode standard», chaque chapitre présente le contenu détaillé immédiatement après l'exposé du problème, puis on trouve une question pour contrôler la matière apprise et finalement le résumé en tant que répétition. Dans le «mode express», l'exposé du problème et le résumé apparaissent en premier. Si les candidats se sentent suffisamment sûrs, ils peuvent sauter les contenus de formation et passer directement au contrôle de la matière apprise. Au cas où l'on n'a pas répondu correctement à une question, on parvient automatique- ment aux contenus détaillés. Dans ces deux modes, un chapitre est considéré comme terminé dès que la question de contrôle a obtenu une réponse correcte. Si le candidat n'a pas répondu juste, une nouvelle question lui sera posée au prochain essai. A l'intention des candidats qui disposent déjà de bonnes connaissances préalables, un «check-up»a été préparé pour chaque leçon. Dès qu'un chapitre a été choisi, deux questions de contrôle sont présentées en «mode examen». Si les deux réponses sont correctes, le chapitre est considéré comme étudié. Pour l'examen final, seuls les brefs résumés des chapitres obligatoires sont déterminants.

\section{Projet pilote d'enseignement en ligne (e-learning)}

L'enseignement en ligne, qui peut être suivi aussi bien en français qu'en allemand, a été testé après sa mise au point par un groupe de médecins-assistants. Formé par l'ASMAC, ce «peer group» a dû traiter tous les chapitres à fond. Il pouvait faire un commentaire en ligne directement après chaque élément d'enseignement. En outre, des entretiens portant sur la qualité ont été menés à l'achèvement de la phase-pilote. On a ensuite revu tout l'enseignement en ligne sur la base des critiques émises en éliminant les points faibles.

\section{Conclusions}

Dix ans après l'entrée en vigueur de la loi sur la radioprotection, la formation postgraduée théorique en radioprotection est devenue plus efficace grâce à des méthodes d'enseignement modernes et elle a été adaptée aux besoins des candidats. La formation postgraduée pratique, dont sont responsables les directeurs des établissements de formation postgraduée clinique, dispose ainsi d'une base solide pour son futur développement. 


\section{Phase test: évaluation des entrevues avec le «peer group»}

Peer group

Le «peer group» était constitué de sept participants (4 femmes et 3 hommes) âgés entre 29 et 37 ans. Tous durent examiner l'ensemble des 160 chapitres de l'enseignement à distance, à savoir tous les chapitres obligatoires et facultatifs, ce qui nécessita en moyenne 17 heures et $\mathbf{4 1}$ minutes par personne (valeur médiane). Les membres du groupe ont ensuite répondu à des questions structurées dans le cadre d'entretiens individuels.

Résultats (aspects techniques)

Comment vous êtes-vous orienté-e dans le système de navigation? $(\mathrm{N}=7)$

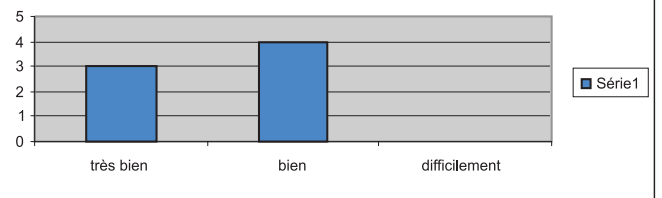

Comment trouvez-vous la présentation de radia.ch (graphisme, mise en page) ? $(\mathrm{N}=7)$

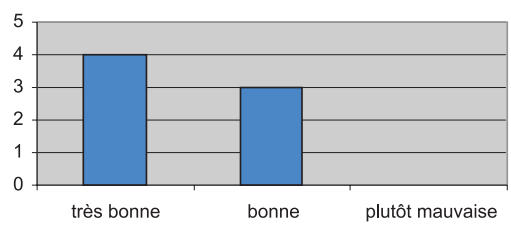

Remarques positives concernant l'enseignement en ligne

Horaire d'étude flexible, rythme d'apprentissage personnel, contrôle personnel par des questions test, bonne vue d'ensemble, possibilité de répéter les leçons, animations, bonne assistance de la part de la firme pnn ag.

\section{Critiques concernant l'enseignement en ligne}

Lenteur d'apparition des pages, imprécision concernant les points importants de l'examen.

Propositions d'amélioration, suggestions de développement

Mise en page (écriture plus foncée, plus d'espace entre les lignes, graphiques plus grands), courriels de rappel avant l'examen, résumé à imprimer, outils pour la préparation de l'examen.

Critiques concernant la formation en radioprotection en général

- Equilibre entre la physique et la médecine;

- complexité de la matière;

- volume de la matière enseignée. 